\title{
Complementary means with respect to a nonsymmetric invariant mean
}

\author{
JANUSZ MATKOWSKI
}

\begin{abstract}
It is known that if a bivariate mean $K$ is symmetric, continuous and strictly increasing in each variable, then for every mean $M$ there is a unique mean $N$ such that $K$ is invariant with respect to the mean-type mapping $(M, N)$, which means that $K \circ(M, N)=$ $K$ and $N$ is called a $K$-complementary mean for $M$ (Matkowski in Aequ Math 57(1):87$107,1999)$. This paper extends this result for a large class of nonsymmetric means. As an application, the limits of the sequences of iterates of the related mean-type mappings are determined, which allows us to find all continuous solutions of some functional equations.
\end{abstract}

Mathematics Subject Classification. Primary 26E60, 39B22, Secondary 39B12.

Keywords. Means, Mean-type mapping, Invariant means, Complementary means, Iteration, Functional equation.

\section{Introduction}

Let $K, M$ and $N$ be bivariate means in an interval $I$. The mean $K$ is called invariant with respect to the mean-type mapping $(M, N): I^{2} \rightarrow I^{2}$, briefly $(M, N)$ - invariant, if $K \circ(M, N)=K$ (cf. [3]). This $K$ is sometimes called the Gauss composition of the means $M$ and $N$ (cf. [2]), and $N$ is referred to as a complementary mean to $M$ with respect to $K$ (briefly, $K$-complementary mean to $M)([3]$, see also [6]).

It is known that, under general conditions, the invariance relation guarantees the convergence of the sequence $\left((M, N)^{n}: n \in \mathbb{N}\right)$ of iterates of the mean-type mapping $(M, N)$ to the mean-type $(K, K)$. This fact has important applications in effectively solving some functional equations.

In [3] it was shown that if the mean $K$ is symmetric, continuous and strictly increasing in each variable, then for every mean $M$ there is a unique $K$-complementary mean. 
The main results of this paper, Theorems 1 and 2, omitting the symmetry condition, give essential generalizations of this result. In particular, Theorem 1 , where the symmetry of $K$ is replaced by the implication " $x<y \Longrightarrow$ $K(x, y) \geq K(y, x)$ for all $x, y \in I$ ", says that for every bivariate mean $M$ in $I$ there is a unique mean $M_{[K]}$ such that $K$ is $\left(M, M_{[K]}\right)$-invariant. Moreover, $M_{[K]}$ is strict or continuous if so is $M$, and both $M$ and $M_{[K]}$ are symmetric if so is $K$. Theorem 2 is a dual counterpart of Theorem 1 . Here the symmetry of $K$ is replaced by the implication " $x<y \Longrightarrow K(x, y) \leq K(y, x)$ for all $x, y \in I$ ". For every mean $M$ it provides a unique mean $M^{[K]}$ such that $K$ is $\left(M^{[K]}, M\right)$-invariant, and has similar additional properties as $M_{[K]}$.

A simple example shows that the assumed implications in Theorems 1 and 2 are essential. Moreover it is shown (Remark 3 and Example 2) that in these results the assumption that $K$ is strictly increasing cannot be replaced by the strictness of $K$.

Applying Theorems 1 and 2 we give conditions under which the sequences of mean-type mappings $\left(M, M_{[K]}\right)$ and $\left(M^{[K]}, M\right)$ converge to $(K, K)$ (Theorem 3 and Theorem 4). This permits, in particular, to determine effectively all continuous functions $F: I^{2} \rightarrow \mathbb{R}$ satisfying each of the functional equations

$$
\begin{aligned}
& F\left(M(x, y), M_{[K]}(x, y)\right)=F(x, y) \quad x, y \in I, \\
& F\left(M^{[K]}(x, y), M(x, y)\right)=F(x, y), \quad x, y \in I .
\end{aligned}
$$

\section{Complementary means}

In the sequel $I \subset \mathbb{R}$ stands for an interval.

A function $M: I \times I \rightarrow I$ is called a mean in $I$, if

$$
\min (x, y) \leq M(x, y) \leq \max (x, y), \quad x, y \in I .
$$

A mean $M$ is called strict, if for $x \neq y$, both these inequalities are sharp; and symmetric, if $M(x, y)=M(y, x)$ for all $x, y \in I$.

Every mean is reflexive, that is $M(x, x)=x$ for all $x \in I$.

Remark 1. If a function $M: I \times I \rightarrow \mathbb{R}$ is reflexive and (strictly) increasing in each of the variables, then it is a (strict) mean in $I$.

Let $K, M, N: I^{2} \rightarrow I$ be means. The mean $K$ is called invariant with respect to the mean-type mapping $(M, N): I^{2} \rightarrow I^{2}$, briefly $(M, N)$-invariant, if $K \circ(M, N)=K$ (cf. [3]). In the case when $K$ is unique, it is sometimes called the Gauss composition of the means $M$ and $N$ (cf. [2]). If $K$ is a unique $(M, N)$-invariant mean we say that $N$ is a complementary mean to $M$ with respect to $K$ (briefly, $N$ is $K$-complementary to $M$ ).

Recall that if $M, N$ are continuous and strict means, then there exists a unique $(M, N)$-invariant mean (cf. [1], also [4], [2], [5]). 
Theorem 1. Let a continuous mean $K: I^{2} \rightarrow I$ be increasing in the first variable and strictly increasing in the second one. Suppose that $K$ satisfies the following condition:

$$
x<y \Longrightarrow K(x, y) \geq K(y, x), \quad x, y \in I .
$$

Then

(i) for every mean $M: I^{2} \rightarrow I$ there is a unique mean $M_{[K]}: I^{2} \rightarrow I$ such that $K$ is $\left(M, M_{[K]}\right)$-invariant, i.e.

$$
K\left(M(x, y), M_{[K]}(x, y)\right)=K(x, y), \quad x, y \in I ;
$$

(ii) if for a symmetric mean $M$, the mean $M_{[K]}$ is symmetric, then $K$ is symmetric;

(iii) if $M$ is strict then so is $M_{[K]}$;

(iv) if $M$ is continuous then so is $M_{[K]}$.

Proof. Take an arbitrary mean $M: I^{2} \rightarrow I$ and $x, y \in I$. We have

$$
\min (x, y) \leq M(x, y) \leq \max (x, y) .
$$

By setting $z=M_{[K]}(x, y)$, the equation (2) becomes

$$
K(M(x, y), z)=K(x, y) .
$$

If $x=y$ then $M(x, y)=K(x, y)=x$ so this equation simplifies to

$$
K(x, z)=x .
$$

Since, by the reflexivity of every mean, $K(x, x)=x$, the strict monotonicity of $K$ in the second variable implies that $M_{[K]}(x, y):=z=x$ is the only solution of this equation.

Assume that $x<y$.

In this case we have

$$
x \leq M(x, y) \leq y .
$$

Define the function $\varphi:[x, y] \rightarrow \mathbb{R}$ by

$$
\varphi(t):=K(M(x, y), t)-K(x, y), \quad t \in[x, y] .
$$

Of course, $\varphi$ is continuous.

For $t=x$, making use first of the inequality $x \leq M(x, y)$ and implication (1), and then from the inequality $M(x, y) \leq y$ and the monotonicity of $K$ in the second variable, we have

$$
\varphi(x)=K(M(x, y), x)-K(x, y) \leq K(x, M(x, y))-K(x, y)<0 .
$$

For $t=y$, the inequality $x \leq M(x, y)$ and the monotonicity of $K$ in the first variable give

$$
\varphi(y)=K(M(x, y), y)-K(x, y) \geq K(x, y)-K(x, y)=0 .
$$


The Darboux property of $\varphi$ implies that there is $z \in[x, y]$ such that $\varphi(z)=0$, i.e. such that

$$
K(M(x, y), z)=K(x, y) .
$$

By the strict monotonicity of $K$ in the second variable, such a $z$ is unique. Setting here $M_{[K]}(x, y):=z$, we get

$$
K\left(M(x, y), M_{[K]}(x, y)\right)=K(x, y) .
$$

Since in the case $y<x$ we can argue similarly, the proof of (i) is complete.

(ii) This is a trivial consequence of (2).

(iii) Assume, on the contrary, that $M_{[K]}$ is not strict, so there are $x_{0}, y_{0} \in I$, $x_{0}<y_{0}$, such that $M_{[K]}\left(x_{0}, y_{0}\right)=x_{0}$ or $M_{[K]}\left(x_{0}, y_{0}\right)=y_{0}$.

In the first case, from (2), we would have

$$
K\left(M\left(x_{0}, y_{0}\right), x_{0}\right)=K\left(M\left(x_{0}, y_{0}\right), M_{[K]}\left(x_{0}, y_{0}\right)\right)=K\left(x_{0}, y_{0}\right),
$$

and, from (1),

$$
K\left(x_{0}, y_{0}\right) \geq K\left(y_{0}, x_{0}\right),
$$

whence $K\left(M\left(x_{0}, y_{0}\right), x_{0}\right) \geq K\left(y_{0}, x_{0}\right)$. But this is a contradiction as $K$ is strictly increasing in the first variable and $x_{0}<M\left(x_{0}, y_{0}\right)<y_{0}$.

Since in the case when $M_{[K]}\left(x_{0}, y_{0}\right)=y_{0}$ we can argue similarly, this completes the proof of (iii).

(iv) We omit the simple argument of this part.

Definition 1. Under the conditions of Theorem 1, the mean $M_{[K]}$ is called $K$-complementary (or $K$-right complementary) to the mean $M$.

Under the conditions of this theorem, part (i) can be strengthened as follows:

Remark 2. For every mean $M: I^{2} \rightarrow I$ there is a unique function $N: I^{2} \rightarrow I$ such that

$$
K(M(x, y), N(x, y))=K(x, y), \quad x, y \in I,
$$

holds and, moreover, $N$ is a mean.

To show that assumption (1) is essential, consider the following

Example 1. Let $I=\mathbb{R}$ and $K: \mathbb{R}^{2} \rightarrow \mathbb{R}$ be a weighted arithmetic mean, i.e. $K(x, y)=a x+(1-a) y$ for some fixed $a \in(0,1)$, and let $M: \mathbb{R}^{2} \rightarrow \mathbb{R}$ be a mean. A simple calculation shows that a function $N: \mathbb{R}^{2} \rightarrow \mathbb{R}$ satisfies (2) if and only if

$$
N(x, y)=\frac{a(x-M(x, y))}{1-a}+y, \quad x, y \in \mathbb{R}
$$


Assume that for every weighted arithmetic mean $M(x, y)=b x+(1-b) y$, $b \in[0,1]$, the function $N$ is a mean in $\mathbb{R}$, that is that

$$
N(x, y)=\frac{a(1-b)}{1-a} x+\left(1-\frac{a(1-b)}{1-a}\right) y, \quad x, y \in \mathbb{R},
$$

is a mean in $\mathbb{R}$. Clearly, $N$ is a mean for every $b \in[0,1]$ if, and only if,

$$
\frac{a(1-b)}{1-a} \leq 1, \quad b \in[0,1] .
$$

Since the function $[0,1] \ni b \longmapsto \frac{a(1-b)}{1-a}$ is decreasing, this inequality holds true iff $\frac{a(1-0)}{1-a} \leq 1$, that is iff

$$
a \leq \frac{1}{2}
$$

We can also argue as follows. The function $N$ given by (3) is a mean for every mean $M$ if and only if

$$
\min (x, y) \leq \frac{a(x-M(x, y))}{1-a}+y \leq \max (x, y), \quad x, y \in \mathbb{R},
$$

or, equivalently, if and only if, for every mean $M$, and for all $x, y \in \mathbb{R}$,

$$
\begin{aligned}
& a x+(1-a) y-(1-a) \max (x, y) \\
& \quad \leq a M(x, y) \leq a x+(1-a) y-(1-a) \min (x, y) .
\end{aligned}
$$

Taking here first $M=\min$ and then $M=\max$ we obtain, respectively, the inequalities

$$
\begin{array}{ll}
a x+(1-a) y \leq a \min (x, y)+(1-a) \max (x, y), & x, y \in \mathbb{R}, \\
(1-a) \min (x, y)+a \max (x, y) \leq a x+(1-a) y, & x, y \in \mathbb{R} .
\end{array}
$$

It is easy to see that each of these inequalities holds, if and only if $a \leq \frac{1}{2}$.

Note that with $K(x, y)=a x+(1-a) y$, the inequality $a \leq \frac{1}{2}$ is equivalent to implication (1).

If $K$ satisfies the "opposite" condition to (1), interchanging the roles of variables in $K$ and applying Theorem 1, we obtain the following

Theorem 2. Let a continuous mean $K: I^{2} \rightarrow I$ be strictly increasing in the first variable and increasing in the second one. Suppose that $K$ satisfies the following condition:

$$
x<y \Longrightarrow K(x, y) \leq K(y, x), \quad x, y \in I .
$$

Then

(i) for every mean $M: I^{2} \rightarrow I$ there is a unique mean $M^{[K]}: I^{2} \rightarrow I$ such that $K$ is $\left(M, M^{[K]}\right)$-invariant, i.e.

$$
K\left(M^{[K]}(x, y), M(x, y)\right)=K(x, y), \quad x, y \in I
$$


(ii) if for some symmetric mean $M$, the mean $M^{[K]}$ is symmetric, then $K$ is symmetric;

(iii) if $M$ is strict then so is $M^{[K]}$;

(iv) if $M$ is continuous then so is $M^{[K]}$.

Definition 2. Under the conditions of Theorem 2, the mean $M^{[K]}$ can be referred to as $K$-complementary (or $K$-left complementary) to the mean $M$.

From Theorems 1 and 2 we obtain the following improvement of Remark 1 in [3]:

Corollary 1. If $K: I^{2} \rightarrow I$ is a continuous strictly increasing in each variable and symmetric mean, then for every mean $M: I^{2} \rightarrow I$ there is a unique function $N: I^{2} \rightarrow I$ such that $K \circ(M, N)=K$, moreover $N$ is a mean and

$$
M_{[K]}=N=M^{[K]} .
$$

We end this section with

Remark 3. In Theorems 1 and 2 (as well as in [3], Remark 1), the strict increasing monotonicity in each variable of the invariant mean $K: I^{2} \rightarrow I$, cannot be weaken by the assumption that $K$ is a strict mean.

To show it consider the following

Example 2. It is known that the contra-harmonic mean $K:(0, \infty)^{2} \rightarrow(0, \infty)$,

$$
K(x, y)=\frac{x^{2}+y^{2}}{x+y},
$$

is not strictly increasing. The symmetry of $K$ implies that condition (1) of Theorem 1 is satisfied. Taking for $M$ the arithmetic mean $A(x, y)=\frac{x+y}{2}$ for $x, y>0$, by a simple calculation, we get

$$
K_{[A]}(x, y)=\frac{x^{2}+y^{2}+\sqrt{2} \sqrt{x^{4}+y^{4}}}{2(x+y)}, \quad x, y>0 .
$$

Of course, $K_{[A]}:(0, \infty)^{2} \rightarrow(0, \infty)$ and $K_{[A]}$ is reflexive, i.e. $K_{[A]}(x, x)=x$; so $K_{[A]}$ is a bivariate pre-mean in $(0, \infty)$. But, as

$$
K_{[A]}(1,10)>11
$$

$K_{[A]}$ is not a mean.

(It can be verified similarly, that $K_{[G]}, K_{[H]}$ where $G$ and $H$ stand, respectively, for the geometric and harmonic mean, are pre-means, but not means.) 


\section{Some applications}

Applying Theorem 1 and the main result of [5] (see also [4]) we obtain the following

Theorem 3. Suppose that a mean $K: I^{2} \rightarrow I$ is continuous and strictly increasing in each variable.

(i) If $K$ satisfies the condition

$$
x<y \Longrightarrow K(x, y) \geq K(y, x), x, y \in I,
$$

then for every continuous and strict mean $M: I^{2} \rightarrow I$, the sequence $\left(\left(M, M_{[K]}\right)^{n}: n \in \mathbb{N}\right)$ of iterates of the mean-type mapping $\left(M, M_{[K]}\right)$ : $I^{2} \rightarrow I^{2}$ converges uniformly on compact sets to the mean-type map $(K, K)$.

(ii) If $K$ satisfies the condition

$$
x<y \Longrightarrow K(x, y) \leq K(y, x), \quad x, y \in I,
$$

then for every continuous and strict mean $M: I^{2} \rightarrow I$, the sequence $\left(\left(M^{[k]}, M\right)^{n}: n \in \mathbb{N}\right)$ of iterates of the mean-type mapping $\left(M^{[k]}, M\right)$ : $I^{2} \rightarrow I^{2}$ converges uniformly on compact sets to the mean-type map $(K, K)$.

Using this result we prove the following

Theorem 4. Let a mean $K: I^{2} \rightarrow I$ be continuous and strictly increasing in each variable and $M: I^{2} \rightarrow I$ be an arbitrary strict and continuous mean.

(i) Suppose that $K$ satisfies the condition

$$
x<y \Longrightarrow K(x, y) \geq K(y, x), x, y \in I .
$$

Then a function $F: I^{2} \rightarrow \mathbb{R}$ continuous at every point of the diagonal $\Delta\left(I^{2}\right):=\{(x, x): x \in I\}$ satisfies the functional equation

$$
F\left(M(x, y), M_{[K]}(x, y)\right)=F(x, y), \quad x, y \in I,
$$

if and only if there is a single variable continuous function $\varphi: I \rightarrow \mathbb{R}$ such that

$$
F(x, y)=\varphi(K(x, y)), \quad x, y \in I .
$$

(ii) Suppose that $K$ satisfies the condition

$$
x<y \Longrightarrow K(x, y) \leq K(y, x), x, y \in I .
$$

Then a function $F: I^{2} \rightarrow \mathbb{R}$ continuous at every point of the diagonal $\Delta\left(I^{2}\right)$ satisfies the functional equation

$$
\begin{gathered}
F\left(M^{[K]}(x, y), M(x, y)\right) \\
=F(x, y), \quad x, y \in I,
\end{gathered}
$$


if and only if there is a single variable continuous function $\varphi: I \rightarrow \mathbb{R}$ such that

$$
F(x, y)=\varphi(K(x, y)), \quad x, y \in I .
$$

Proof. Assume first that $F: I^{2} \rightarrow \mathbb{R}$ is continuous on the diagonal $\Delta\left(I^{2}\right)$ and satisfies equation (4), that is

$$
F \circ\left(M, M_{[K]}\right)=F .
$$

Hence, by induction,

$$
F=F \circ\left(M, M_{[K]}\right)^{n}, \quad n \in \mathbb{N},
$$

where $\left(M, M_{[K]}\right)^{n}$ is the $n$th iterate of $\left(M, M_{[K]}\right)$. By Theorem 3 the sequence of mean-type mappings $\left(M, M_{[K]}\right)^{n}$ converges to the mean-type mapping $(K, K): I^{2} \rightarrow I^{2}$; that is

$$
\lim _{n \rightarrow \infty}\left(M, M_{[K]}\right)^{n}(x, y)=(K(x, y), K(x, y)), \quad(x, y) \in I^{2} .
$$

Since $(K(x, y), K(x, y))$ belongs to the diagonal $\Delta\left(I^{2}\right)$ for every $(x, y) \in I^{2}$, by (5) and the continuity of $F$ on $\Delta\left(I^{2}\right)$ implies that for every $(x, y) \in I^{2}$,

$$
\begin{aligned}
F(x, y) & =\lim _{n \rightarrow \infty} F\left(\left(M, M_{[K]}\right)^{n}(x, y)\right)=F\left(\lim _{n \rightarrow \infty}\left(M, M_{[K]}\right)^{n}(x, y)\right) \\
& =F(K(x, y), K(x, y)) .
\end{aligned}
$$

Setting

$$
\varphi(t):=F(t, t), \quad t \in I,
$$

we conclude that $F(x, y)=\varphi(K(x, y))$ for all $(x, y) \in I^{2}$.

To prove the converse implication, take an arbitrary function $\varphi: I \rightarrow \mathbb{R}$ and put $F:=\varphi \circ K$. Then, for all $x, y \in I$, making use of the $K$-invariance with respect to $M, M_{[K]}$, we have

$$
\begin{aligned}
F\left(M(x, y), M_{[K]}(x, y)\right) & =(\varphi \circ K)\left(M(x, y), M_{[K]}(x, y)\right) \\
& =\varphi\left(K\left(M(x, y), M_{[K]}(x, y)\right)\right)=\varphi(K(x, y)) \\
& =F(x, y),
\end{aligned}
$$

which completes the proof of (i).

We omit the similar proof of (ii).

\section{Acknowledgements}

The author would like to thank the Anonymous Referee who provided useful and detailed comments on a previous version of the manuscript, and especially for the question inspiring Remark 3. 
Open Access. This article is licensed under a Creative Commons Attribution 4.0 International License, which permits use, sharing, adaptation, distribution and reproduction in any medium or format, as long as you give appropriate credit to the original author(s) and the source, provide a link to the Creative Commons licence, and indicate if changes were made. The images or other third party material in this article are included in the article's Creative Commons licence, unless indicated otherwise in a credit line to the material. If material is not included in the article's Creative Commons licence and your intended use is not permitted by statutory regulation or exceeds the permitted use, you will need to obtain permission directly from the copyright holder. To view a copy of this licence, visit http:// creativecommons.org/licenses/by/4.0/.

Publisher's Note Springer Nature remains neutral with regard to jurisdictional claims in published maps and institutional affiliations.

\section{References}

[1] Borwein, J.M., Borwein, P.M.: Pi and the AGM. Wiley, Hoboken, NJ (1986)

[2] Daróczy, Z., Páles, Z.: On functional equations involving means. Publ. Math. Debrecen 62, 363-377 (2003)

[3] Matkowski, J.: Invariant and complementary quasi-arithmetic means. Aequ. Math. 57(1), 87-107 (1999)

[4] Matkowski, J.: Iterations of mean-type mappings and invariant means. In: European conference on iteration theory (Muszyna-Złockie, 1998). Ann. Math. Sil. No. Vol. 13, pp. 211-226 (1999)

[5] Matkowski, J.: Iterations of the mean-type mappings and uniqueness of invariant means. Ann. Univ. Sci. Budapest. Sect. Comput. 41, 145-158 (2013)

[6] Toader, G., Costin, I.: Means in Mathematical Analysis. Bivariate Means. Mathematical Analysis and Its Applications. Academic Press, London (2018)

Janusz Matkowski

Institute of Mathematics

University of Zielona Góra

Szafrana 4A

PL65-516 Zielona Góra

Poland

e-mail: J.Matkowski@wmie.uz.zgora.pl

Received: June 22, 2020

Revised: August 9, 2021

Accepted: August 12, 2021 